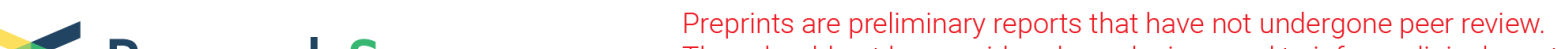 $\begin{array}{ll}\text { Research Square } & \text { They should not be considered conclusive, used to inform clinical practice, } \\ \text { or referenced by the media as validated information. }\end{array}$
}

\section{A Multilevel Systemic Pan-cancer Analysis: Collagen Triple Helix Repeat Containing-1 is a Potential Target for Tumor Immunotherapy}

\section{Jiangtao Zhang}

Department of Gastrointestinal and Gland Surgery, First Affiliated Hospital of Guangxi Medical University

\section{Xianghua Wu ( $\nabla$ wuxianghua@stu.gxmu.edu.cn )}

Department of Gastrointestinal and Gland Surgery, First Affiliated Hospital of Guangxi Medical University

\section{Huichao Ruan}

Department of Gastrointestinal and Gland Surgery, First Affiliated Hospital of Guangxi Medical University

\section{Changli Wang}

Department of Nutrition, Second Affiliated Hospital of Guangxi Medical University

\section{Rong qiang Yang}

Department of Gastrointestinal and Gland Surgery, First Affiliated Hospital of Guangxi Medical University xiaobing Fu

Department of Gastrointestinal and Gland Surgery, First Affiliated Hospital of Guangxi Medical University

\section{Research Article}

Keywords: Pan cancer, prognosis, immune checkpoint, CTHRC1

Posted Date: November 10th, 2021

DOI: https://doi.org/10.21203/rs.3.rs-1016486/v1

License: (c) (1) This work is licensed under a Creative Commons Attribution 4.0 International License. Read Full License 


\section{Abstract}

Background: Cancer is one of the leading causes of pathological death in humans. Although CTHRC1 is a prooncogene highly expressed in a variety of tumor tissues, the specific biological mechanisms of CTHRC1 involvement in cancer development need to be elucidated.

Methods: In the present study, nine online bioinformatics databases were employed to explore the potential prognostic and grading value of CTHRC1 in generalized cancer as well as its potential role in regulating tumor immunity.

Results: Data from GEPIA2.0, Oncomine, TNMplot, Kaplan-Meier Plotter and TISIDB database had consistently demonstrated that CTHRC1 was associated with the expression, prognosis and typing in most cancer tissues. Cbioportal and SMART analysis revealed that genomic changes and methylation of CTHRC1 in most tumor tissues. Finally, Sangerbox and TIMER database analysis suggested that CTHRC1 was involved in the changes of immune cell components in tumor immune microenvironment, with certain heterogeneity. Meanwhile, CTHRC1 was correlated with TMB, MSI, neoantigen and tumor immune checkpoint, especially CD276.

Conclusion: $C T H R C 1$ had the potential as a prognostic and grading molecular marker for pan-cancer. And CTHRC1-related targeting agents may be a novel breakthrough in tumor immunotherapy.

\section{Introduction}

According to the World Health Organization (WHO), cancer will be the deadliest diseases in the 21st century[1]. Despite various tumor treatment methods are actively pursued, several issues remain. Such as poor tumor results, rapid disease progression, and high mortality. the mobilization of the immune system and enhancement of antitumor immunity are the main principles of cancer immunotherapy. As against traditional treatment, immunotherapy is characterized with high specificity and low side effects. The development of cancer immunotherapy represents a novel direction of cancer treatment

Collagen triple helix repeat containing-1(CTHRC1) is first discovered in injured arteries of rats, which encodes protein that belongs to a highly conserved glycosylated protein[2]. In the field of tumor, CTHRC1 activates invasion and migration of gastric cancer (GC) cell lines SGC7901 and HGC27 via hiF-1/CXCR4 axis[3]. He et al. report that high expression of $C T H R C 1$ protein in lung cancer tissues, which affects lung cancer cells' invasion and metastasis by acting on MMP7/MMPQ[4]. Zheng et al. found that CTHRC1 promotes HeLa cell growth via activating the Wnt/PCP signaling pathway[5]. Over-expression of CTHRC1 is found to be a marker of worse prognosis in several different malignancies, including colorectal cancer[6], lung cancer[7], gastric cancer[8] and liver cancer[9]. Those results implicate CTHRC1 is not only involved in regulating tumor cells growth, proliferation and apoptosis via multiple pathways, but also has the potential as a prognostic marker for pan-cancer. 
The tumor microenvironment (TME) acts a pivotal part in the occurrence, development, metastasis and drug sensitivity of tumors. CTHRC1 can promote M2 macrophage polarization via STAT6 signaling pathway in ovarian cancer tissues[10]. CTHRC1 regulates the integrin-akt signaling pathway to promote M2 macrophage polarization and invasion and metastasis of endometrial carcinoma cells[11]. CTHRC1overexpressed patients with urological cancers have a poor prognosis in part because of alterations in TME[12]. Above studies suggest that $C T H R C 1$ plays a certain role in regulating tumor immunity. However, there are no published reports focusing on the prognosis and immunomodulatory mechanism of CTHRC1 in pan-cancer.

Here, the expression differences, prognostic potential, immunotyping and molecular typing of CTHRC1 in human cancer were comprehensively investigated by multiple online analysis tools based The Cancer Genome Atlas (TCGA) resources. Next, Understanding CNV and methylation information of CTHRC1 in tumor tissues, and its role in tumor immunity through comprehensive and multi-dimensional analysis. Overall, the main objective of this study was to evaluate potential value of $C T H R C 1$ in immunotherapy of malignant tumors.

\section{Methods}

\subsection{Four databases define CTHRC1 transcript levels in various tumor tissues}

GEPIA2.0 database (http://gepia2.cancer-pku.cn/\#index) is developed based on RNA-seq data from thousands of tumor and normal samples of TCGA and GTEx[13]. The TIMER

(https://cistrome.shinyapps.io/timer/) is a web-based public tool based on TCGA[14]. The Oncomine database ( https://software.oncomine.com ) is a public open database, which incorporates thousands of microarray datasets from human tumors[15]. The screening condition was that $P<0.001$, Change $=2$. The TNMplot database (https://tnmplot.com/analysis/)belongs to a freely available bioinformatics website, which contains mainly RNA-seq data from TCGA, GTEX and TARGET[16]. Above databases was used to investigate the differential expression of CTHRC1 mRNA between tumor and normal tissue, as shown by box plots.

\subsection{Two databases define the prognostic value of CTHRC1 in human cancers}

GEPIA2.0 can be used not only for the analysis of single gene expression differences, but also for the prognostic value of gene in cancers. Input CTHRC1, the selection condition: $\mathrm{P}<0.05$, Group Cutoff: Media. K-M plotter (http://kmplot.com/analysis/index.php?p=background) is a classic website focused on cancer prognosis and contains RNA-seq and gene chip data for 21 cancers[17]. Using microarray data from this website. we analyzed the prognostic value of $C T H R C 1$ in some cancers. 


\subsection{Identify CTHRC1 mRNA levels in tumors immunity and molecular typing by TISIDB}

TISIDB (http://cis.hku.hk/TISIDB/) is a powerful tumor immunity database developed based on TCGA resources[18]. Enter $C T H R C 1$ on the home page and select the Subtype module. Identify the corresponding cancer type and output the corresponding picture.

\subsection{Two databases were used to analyze CTHRC1 CNV and methylation in human cancer tissues}

Cbioportal (http://www.cbioportal.org/) is a bioinformatics analysis tool developed based on TCGA, ICGC and GEO data resources, which can study the mutation and copy number variation of CTHRC1 in different cancer types[19]. At the same time, it can also be combined with clinical data to investigate the relevance between $C T H R C 1 \mathrm{CNV}$ and the prognosis of cancer patients. Input $C T H R C 1$, and select the consistent sample TCGA Pan-Cancer Atlas. The Oncoprint module presents details of genomic alterations in CTHRC1 in cancer. Cancer summar Types survival module is used to understand the association of the CTHRC1 gene alteration with the prognosis. SMART (http://www.bioinfo-zs.com/smartapp/) is an online website built based on TCGA tumor methylation data, which can the relationship between different methylation sites of genes and expression differences, prognosis and grade[20]. On the home page, CTHRC1 was entered and the Click to check CpG-aggregated methylation module was selected to visually analyze CTHRC1 methylation in pantothenic cancer. Then, the prognostic module was selected to view the prognosis of different methylation sites in a variety of cancers.

\subsection{Two databases jointly analyze the link between CTHRC1 and the tumor immune microenvironment}

Sangerbox (http://sangerbox.com/Tool), which is a medical bioinformatics platform built using TCGA resources[21]. Enter CTHRC1 to view links to TMB, MSI, neoantigens, and immune checkpoints in different cancer types. Gene module of The TIMER database was additionally used to understand the link between $C T H R C 1$ and a variety of immune cell numbers in cancer tissues.

\section{Statistical Analysis}

All statistical methods were provided by the corresponding website. Statistical significance was considered at $\mathrm{P}<0.05$.

\section{Results}

\subsection{Differential expression of CTHRC1 in non-tumor and tumor tissues}


In a first step, significant difference was observed for expression of CTHRC1 mRNA in non-tumor and tumor tissues. By analyzing the gene chip data of Oncomine, expression levels of CTHRC1 are high in most tumor tissues, consisting of brain and CNS cancer, breast cancer, cervical cancer, colorectal cancer, esophageal cancer, gastric cancer, head and neck cancer, kidney cancer, leukemia, liver cancer, lung cancer, lymphoma, melanoma, ovarian cancer, pancreatic cancer and prostate cancer. In TIMER database, Compared with the normal counterparts, the transcript level of CTHRC1 was over-expressed in most cancer types, including BLCA (bladder urothelial carcinoma), BRCA (breast invasive carcinoma), CHOL (cholangio carcinoma), COAD (colon adenocarcinoma), HNSC (head and neck cancer), ESCA (esophageal carcinoma), KIRC (kidney renal clear cell carcinoma), KIRP (kidney renal papillary carcinoma), LIHC (liver hepatocellular carcinoma), LUAD (lung adenocarcinoma), LUSC (lung squamous cell carcinoma), PRAD (prostate adenocarcinoma), READ (rectum adenocarcinoma), STAD (stomach adenocarcinoma), THCA (thyroid carcinoma) and UCEC(uterine corpus endometrial carcinoma). Most of the results of the UALCAN analysis bear a close resemblance data from the TIMER database. And CTHRC1 mRNA was also upregulated in CESC (cervical squamous cell carcinoma and endocervical adenocarcinoma), GBM (glioblastoma multiforme), KICH (kidney chromophobe), SARC (sarcoma) and THYM (thymoma). In TNMplot, CTHRC1 mRNA was also upregulated in BLCA, BRCA, COAD, ESCA, LIHC, LUAD, LUSC, OV, PAAD (pancreatic adenocarcinoma), PRAD, READ, KIRC, KIRP, SKCM (skin cutaneous melanoma), STAD, TGCT (testicular germ cell tumors), UCS (uterine carcinosarcoma), ACC (adrenocortical carcinoma), LAML (acute myeloid leukemia) and UCEC. The above results tentatively proposed that CTHRC1 was an oncogene and had potential as a diagnostic marker for pan-cancer.

\subsection{CTHRC1 and prognosis of cancer patients}

Our study next found that over-expression of $C T H R C 1$ affects a poor prognosis of patients based on GEPIA2.0 (TCGA RNA-seq data) and KM-plotter (gene chip data). Detailed results are presented in the following sets of data. In GEPIA2.0, ACC [OS: $H R=4.2, P=0.00039$, DFS: $H R=5, P<5.6 e-06$ ]; COAD [OS: $H R=1.8, P=0.018$, DFS: $H R=1.8, P=0.015]$; $K I R P$ [OS: $H R=2, P=0.029$, DFS: $H R=1.9, P=0.027$ ]; $B L C A$ [OS: $H R=1.4, P=0.045$ ]; $L G G$ [OS: $H R=1.6, P=0.015] ; ~ L I H C$ [OS: $H R=1.6, P=0.0094] ; S A R C$ [OS: $H R=1.8$, $P=0.0059$ ]; STAD [OS: $H R=1.4, P=0.049$ ]; $K I C H$ [DFS: $H R=4.4, P=0.04$ ]; $K I R C$ [DFS: $H R=2.2, P<2.1 \mathrm{e}-05$ ]; PRAD [DFS: $H R=1.8, P=0.0064]$. In KM-plotter, OV [OS: $H R=1.41, P=0.000087$, PFS: $H R=1.41, P=$ $0.00028, P P S: H R=1.3, P=0.032$ ]; $B R C A$ [DMFS: $H R=1.32, P=0.025]$. This result implies that $C T H R C 1$ has the potential to be used as a prognostic marker in pan-cancer.

\subsection{Relative expression levels of CTHRC1 in different tumor subtypes (immune and molecular subtypes)}

Using the Subtype module of the TISIDB database, we found that $C T H R C 1$ transcript levels significantly varied in immune subtypes [ $\mathrm{C} 1$ (wound healing), C2 (IFN-gamma dominant), C3 (inflammatory), C4 (lymphocyte depleted), C5 (immunologically quiet) and C6 (TGF-b dominant)] of multiple tumors, which include BLCA, BRCA, COAD, KIRP, KICH, KIRC, LIHC, SARC, PRAD, OV and LGG. CTHRC1 was not always highly expressed in a single immune subtype. In more detail, CTHRC1 showed the highest expression in C1 subtype of three distinct tumors, which include KIRC, LIHC and SARC. Highest expression in C2 
subtype, which include BLCA, KIRP and PRAD. Highest expression in C3 subtype, which include KICH, OV and LGG. Highest expression in C4 subtype, which include BRCA and COAD. Furthermore, CTHRC1 expression is also differentially expressed in molecular subtypes of multiple tumors, which include BLCA, BRCA, COAD, KIRP, KICH, KIRC, LIHC, SARC, PRAD, OV and LGG. The above analysis indicated that CTHRC1 has a diagnostic index as a classification of pan-cancer.

\subsection{CTHRC1 CNV and Methylation in tumors}

The use of cBioPortal database was used to analyse the uniform sample (TCGA, PanCancer Atlas). CTHRC1 amplification (AMP) was the most prevalent actionable alteration in multiple tumors, consisting of OV, BLCA, BRCA, LIHC, PRAD, STAD, SARC, COAD, KICH, LGG and KIRC. And CTHRC1 CNV alteration was a high risk factor in cancer patients, consisting of OV, LGG, SARC, PRAD and KIRP. For instance, the group of $C T H R C 1 \mathrm{CNV}$ alteration had a significantly poorer OS, PFS and DSS. More detailed data are shown in (Figure 4). DNA methylation was considered an essential factor impacting on the occurrence and progression of tumor. Currently, there is a paucity of research on the relationship between abnormal methylation of $C T H R C 1$ gene and tumors. Thus, through the analysis results of the SMART database, we demonstrated that abnormal methylation of $C T H R C 1$ gene in a variety of tumor tissues, including BLCA, BRCA, CESC, COAD, ESCA, HNSC, KIRC, LIHC, LUAD, LUSC, READ, THCA and UCEC. Also, we found that CTHRC1 hypermethylation was generally linked to good prognosis in BLCA, BRCA, HNSC, KIRC and UCEC. More detailed results are shown in (Figure 5).

\subsection{CTHRC1 and human cancers immune microenvironment}

The TIMER database found a link between CTHRC1 transcript levels and immune cell numbers in a variety of cancer types. For example, a significant positive association was observed between CTHRC1 and multiple immune cell types such as B cells, CD $4+T$ cells CD $8+T$ cells, neutrophils, macrophages and dendritic cells in BRCA. An additional thing we noticed was that $C T H R C 1$ was not always related to specific immune cell species. Like, CTHRC1 has the largest correlation coefficient with B cells in ten cancer types. Having the closest association with $\mathrm{CD} 4+\mathrm{T}$ cells in eight cancer types. Having the largest correlation coefficient with CD8+T cells in ten cancer types. Having the closest association with macrophages in ten cancer types. Having the closest association with neutrophils in ten cancer types. And having the closest association with dendritic cells in twelve cancer types. This suggests that CTHRC1 exerts different immune effects in different types of tumor tissues. The sangerbox platform analyzed 47 immune checkpoint genes and found a positive correlation between CTHRC1 and immune checkpoint genes in most tumor tissues. In addition, there was a significant positive correlation between CTHRC1 and CD276 in most tumor tissues. TMB, MSI and neoantigens were important evaluation metric of immunotherapy efficacy. There was a positive correlation between CTHRC1 with TMB in COAD, LAML, LGG, LUAD, PRAD, SARC and THYM, and a negative correlation between CTHRC1 with TMB in GBM, LIHC and UCEC. MSI and neoantigens exhibited the closest linkage with CTHRC1. In particular, UCEC patients 
with high CTHRC1 expression tend to show negative scores of TMB, MSI and neoantigens. This suggests that UCEC patients with high CTHRC1 expression have poor immune efficacy.

\section{Discussion}

An extracellular matrix protein encoded by $C T H R C 1$ was integral components of the immune microenvironment. Extracellular matrix (ECM) had been shown to be involved in tumor development via biological pathways, including activating intracellular signaling of cell cycle progression, inhibiting the function of tumor suppressor factors, participating in the invasion and metastasis of cancer cells, tumor suppressor gene mutation and evasion of immune surveillance, etc[22, 23]. Actually, studies have found that CTHRC1 is involved in the progression of human cancer[24]. However, the specific mechanism of CTHRC1 regulates tumor immune microenvironment remains unclear.

First step in this investigation, bioinformatics analysis based on four databases suggests that $C T R H C 1$ is highly expressed in various tumor tissues. It was preliminarily suggested that $C T H R C 1$ is a protooncogene involved in the progression of tumors. Next, GEPIA2.0 and KM-plotter databases found that high expression of CTHRC1 was a negative factor in 10 cancers, implicating CTHRC1 can be served as a pan-cancer diagnostic and prognostic biomarker. The correct classification of cancer patients is clinically conducive to selecting appropriate therapeutic regimens. For most cancers except STAD and ACC, CTHRC1 transcription levels were different in immune and molecular subtypes, which suggested that CTHRC1 may be a novel marker for the diagnosis of pan-cancer and participates in the regulation of tumor immunity. Oncogenic transformation always occurs along with genomic alterations. Liu et al. reported amplification of the levels of CTHRC1 drives proliferation and invasion of oral squamous cell carcinoma[25]. We demonstrated that CTHRC1 was shown to undergo some amplification in most cancers, with the most significant in OV. CTHRC1 gene amplification is a prevalent phenomenon in the vast majority of tumor types. And altered group often implies a worse prognosis in OV, SARC, LGG, KIRP and PRAD. Aberrant DNA methylation is one of the important characteristics of cancer epigenetics. Early research found that $C T H R C 1$ gene demethylation increases expression and is associated with cell line growth and invasive abilities in gastric cancer[26]. As shown in the present study, the methylation level of distinct sites of $C T H R C 1$ gene was significantly lower in most tumor tissues than in neighboring nontumor tissue samples. And CTHRC1 methylation levels are closely related to patient prognosis in BLCA, BRCA, HNSC, KIRC and UCEC. The results provide a deeper theoretical basis for relevant research of cancers epigenetics.

More recently, it has been reported that tumor infiltrating lymphocytes (TILs) could be used as an indicator of evaluating the prognosis and treatment efficacy for cancer patients[27, 28]. Our study suggested that CTHRC1 mRNA levels were closely linked to the number of immune cells and played a critical role in regulating TME. Specifically, a significant positive association was observed between CTHRC1 and number of macrophages in BLCA, BRCA, COAD and STAD. Interestingly, The most strongly positive relationship between CTHRC1 and number of dendritic cells in KICH, KIRP, PRAD and SARC. Thus, this suggests that $C T H R C 1$ can participate in the regulation of tumor microenvironment immune status. 
Noteworthy, the following phenomenon could have contributed to the poor prognosis of cancer patients. First, CTHRC1 has a correlation with the number of macrophages in most tumor tissues. There is a report that macrophages are involved in the process of tumor immune escape[29]. Second, CTHRC1 has a correlation with multiple types of immune checkpoints in most tumor tissues. For example, CTHRC1 has a positive correlation with PDCD1 in PRAD, which is similar to the results of a previous work[30].

Clinically, PD-1 and CTLA4 targeting have already been applied extensively in treatment of cancer[31]. TMB is a promising cancer predictive biomarker, and has significant significance in guiding immune checkpoint inhibitor (ICI) treatments[32, 33]. MSI also be an independent prognostic biomarker of cancers[34-36]. In this current study, the association of CTHRC1 with tumor mutation burden (TMB) and microsatellite instability (MSI) also provided strong evidence that CTHRC1 was involved in the regulation of TME in human cancers. Particularly, CTHRC1 showed a negative correlation with TMB, MSI and neoantigens in UCEC. This may be the reason why some patients with UCEC do not respond well to ICI treatment. Future targeting of $C T H R C 1$ in combination with PDCD1 inhibitors will be a novel direction for UCEC patients.

\section{Conclusion}

Altogether, our study is the first to systematically investigated the link between CTHRC1 and pan-cancer at the mRNA level, CNV level and methylation level as well as prognostic effects by bioinformatics methods. And the study demonstrated that CTHRC1 is an unfavorable prognostic factor in a variety of cancers with a link to alterations in the immune microenvironment. However, there exists some limitations in the present study. First of all, the quantity and quality of public data resources, screening conditions and statistical methods can affect the authenticity and rigor of research. Validation by in vitro and in vivo experiments and pathological specimens is required. In addition, we lack immunological experiments to prove that $C T H R C 1$ has immunomodulatory ability in cancers. Prospective studies of $C T H R C 1$ targeting and the tumor immune microenvironment may help provide a clear anticancer strategy in the future.

\section{Declarations}

\section{Ethics approval and consent to participate}

Not applicable.

\section{Consent for publication}

Not applicable.

\section{Declaration of Competing Interest}

The authors declare that there is no conflicts of interest.

\section{Acknowledgements}


None.

\section{Funding}

The present study was supported by the National Natural Science Foundation of China (No. 81260341) and the Guangxi Natural Science Foundation Program (No. 2017JJA10173).

\section{Availability of data and materials}

All data is provided by the sites mentioned in this article.

\section{Authors' contributions}

WXH and ZJT: conceptualization; FXB and YRQ: data curation and methodology; RHZ and WCL: data analysis and visualization; WXH and ZJT: writing, review, and editing. All authors read and approved the final manuscript.

\section{References}

1. Bray F, Ferlay J, Soerjomataram I, Siegel RL, Torre LA, Jemal A. Global cancer statistics 2018 : GLOBOCAN estimates of incidence and mortality worldwide for 36 cancers in 185 countries. CA: a cancer journal for clinicians. 2018;68(6):394-424.

2. Pyagay $P$, Heroult $M$, Wang Q, Lehnert W, Belden J, Liaw L, Friesel RE, Lindner V. Collagen triple helix repeat containing 1 , a novel secreted protein in injured and diseased arteries, inhibits collagen expression and promotes cell migration. Circulation research. 2005;96(2):261-8.

3. Ding X, Huang R, Zhong Y, Cui N, Wang Y, Weng J, Chen L, Zang M. CTHRC1 promotes gastric cancer metastasis via HIF-1a/CXCR4 signaling pathway. Biomedicine \& pharmacotherapy = Biomedecine \& pharmacotherapie. 2020;123:109742.

4. He W, Zhang H, Wang Y, Zhou Y, Luo Y, Cui Y, Jiang N, Jiang W, Wang H, Xu D, Li S, Wang Z, Chen Y, Sun Y, Zhang Y, Tseng HR, Zou X, Wang L, Ke Z. CTHRC1 induces non-small cell lung cancer (NSCLC) invasion through upregulating MMP-7/MMP-9. BMC cancer. 2018;18(1):400.

5. Zheng M, Zhou Q, Liu X, Wang C, Liu G. CTHRC1 overexpression promotes cervical carcinoma progression by activating the Wnt/PCP signaling pathway. Oncology reports. 2019;41(3):1531-8.

6. Ni S, Ren F, Xu M, Tan C, Weng W, Huang Z, Sheng W, Huang D. CTHRC1 overexpression predicts poor survival and enhances epithelial-mesenchymal transition in colorectal cancer. Cancer medicine. 2018;7(11):5643-54.

7. Chen Y, Sun Y, Cui Y, Lei Y, Jiang N, Jiang W, Wang H, Chen L, Luo J, Chen Y, Tang K, Zhou C, Ke Z. High CTHRC1 expression may be closely associated with angiogenesis and indicates poor prognosis in lung adenocarcinoma patients. Cancer cell international. 2019;19:318.

8. Mei D, Zhu Y, Zhang L, Wei W. The Role of CTHRC1 in Regulation of Multiple Signaling and Tumor Progression and Metastasis. Mediators of inflammation. 2020;2020:9578701. 
9. Zhou H, Su L, Liu C, Li B, Li H, Xie Y, Sun D. CTHRC1 May Serve As A Prognostic Biomarker For Hepatocellular Carcinoma. OncoTargets and therapy. 2019;12:7823-31.

10. Bai Y, Yin K, Su T, Ji F, Zhang S. CTHRC1 in Ovarian Cancer Promotes M2-Like Polarization of TumorAssociated Macrophages via Regulation of the STAT6 Signaling Pathway. OncoTargets and therapy. 2020;13:5743-53.

11. Li LY, Yin KM, Bai YH, Zhang ZG, Di W, Zhang S. CTHRC1 promotes M2-like macrophage recruitment and myometrial invasion in endometrial carcinoma by integrin-Akt signaling pathway. Clinical \& experimental metastasis. 2019;36(4):351-63.

12. Zhou F, Shen D, Xiong Y, Cheng S, Xu H, Wang G, Qian K, Ju L, Zhang X. CTHRC1 Is a Prognostic Biomarker and Correlated With Immune Infiltrates in Kidney Renal Papillary Cell Carcinoma and Kidney Renal Clear Cell Carcinoma. Frontiers in oncology. 2020;10:570819.

13. Tang Z, Kang B, Li C, Chen T, Zhang Z. GEPIA2: an enhanced web server for large-scale expression profiling and interactive analysis. Nucleic acids research. 2019;47(W1):W556-w60.

14. Li T, Fan J, Wang B, Traugh N, Chen Q, Liu JS, Li B, Liu XS. TIMER: A Web Server for Comprehensive Analysis of Tumor-Infiltrating Immune Cells. Cancer research. 2017;77(21):e108-e10.

15. Rhodes DR, Kalyana-Sundaram S, Mahavisno V, Varambally R, Yu J, Briggs BB, Barrette TR, Anstet MJ, Kincead-Beal C, Kulkarni P, Varambally S, Ghosh D, Chinnaiyan AM. Oncomine 3.0: genes, pathways, and networks in a collection of 18,000 cancer gene expression profiles. Neoplasia (New York, NY). 2007;9(2):166-80.

16. Bartha Á, Győrffy B. TNMplot.com: A Web Tool for the Comparison of Gene Expression in Normal, Tumor and Metastatic Tissues. International journal of molecular sciences. 2021;22(5).

17. Győrffy B. Survival analysis across the entire transcriptome identifies biomarkers with the highest prognostic power in breast cancer. Computational and structural biotechnology journal. 2021;19:4101-9.

18. Ru B, Wong CN, Tong Y, Zhong JY, Zhong SSW, Wu WC, Chu KC, Wong CY, Lau CY, Chen I, Chan NW, Zhang J. TISIDB: an integrated repository portal for tumor-immune system interactions.

Bioinformatics (Oxford, England). 2019;35(20):4200-2.

19. Gao J, Aksoy BA, Dogrusoz U, Dresdner G, Gross B, Sumer SO, Sun Y, Jacobsen A, Sinha R, Larsson E, Cerami E, Sander C, Schultz N. Integrative analysis of complex cancer genomics and clinical profiles using the cBioPortal. Science signaling. 2013;6(269):pl1.

20. Li Y, Ge D, Lu C. The SMART App: an interactive web application for comprehensive DNA methylation analysis and visualization. Epigenetics \& chromatin. 2019;12(1):71.

21. Wang D, Wang Y, Zou X, Shi Y, Liu Q, Huyan T, Su J, Wang Q, Zhang F, Li X, Tie L. FOX01 inhibition prevents renal ischemia-reperfusion injury via cAMP-response element binding protein/PPAR-Y coactivator-1a-mediated mitochondrial biogenesis. British journal of pharmacology. 2020;177(2):432-48.

22. Mohan V, Das A, Sagi I. Emerging roles of ECM remodeling processes in cancer. Seminars in cancer biology. 2020;62:192-200. 
23. Najafi M, Farhood B, Mortezaee K. Extracellular matrix (ECM) stiffness and degradation as cancer drivers. Journal of cellular biochemistry. 2019;120(3):2782-90.

24. Sial N, Ahmad M, Hussain MS, Iqbal MJ, Hameed $Y$, Khan M, Abbas M, Asif R, Rehman JU, Atif M, Khan MR, Hameed Z, Saeed H, Tanveer R, Saeed S, Sharif A, Asif HM. CTHRC1 expression is a novel shared diagnostic and prognostic biomarker of survival in six different human cancer subtypes. Scientific reports. 2021;11(1):19873.

25. Liu G, Sengupta PK, Jamal B, Yang HY, Bouchie MP, Lindner V, Varelas X, Kukuruzinska MA. Nglycosylation induces the CTHRC1 protein and drives oral cancer cell migration. The Journal of biological chemistry. 2013;288(28):20217-27.

26. Wang P, Wang YC, Chen XY, Shen ZY, Cao H, Zhang YJ, Yu J, Zhu JD, Lu YY, Fang JY. CTHRC1 is upregulated by promoter demethylation and transforming growth factor- $\beta 1$ and may be associated with metastasis in human gastric cancer. Cancer science. 2012;103(7):1327-33.

27. Denkert C, von Minckwitz G, Darb-Esfahani S, Lederer B, Heppner BI, Weber KE, Budczies J, Huober J, Klauschen F, Furlanetto J, Schmitt WD, Blohmer JU, Karn T, Pfitzner BM, Kümmel S, Engels K, Schneeweiss A, Hartmann A, Noske A, Fasching PA, Jackisch C, van Mackelenbergh M, Sinn P, Schem C, Hanusch C, Untch M, Loibl S. Tumour-infiltrating lymphocytes and prognosis in different subtypes of breast cancer: a pooled analysis of 3771 patients treated with neoadjuvant therapy. The Lancet Oncology. 2018;19(1):40-50.

28. Linette GP, Carreno BM. Tumor-Infiltrating Lymphocytes in the Checkpoint Inhibitor Era. Current hematologic malignancy reports. 2019;14(4):286-91.

29. Liu C, Yao Z, Wang J, Zhang W, Yang Y, Zhang Y, Qu X, Zhu Y, Zou J, Peng S, Zhao Y, Zhao S, He B, Mi Q, Liu X, Zhang X, Du Q. Macrophage-derived CCL5 facilitates immune escape of colorectal cancer cells via the p65/STAT3-CSN5-PD-L1 pathway. Cell death and differentiation. 2020;27(6):1765-81.

30. Zhou Q, Xiong W, Zhou X, Gao RS, Lin QF, Liu HY, Li JN, Tian XF. CTHRC1 and PD-1/PD-L1 expression predicts tumor recurrence in prostate cancer. Molecular medicine reports. 2019;20(5):4244-52.

31. Porcu M, De Silva P, Solinas C, Battaglia A, Schena M, Scartozzi M, Bron D, Suri JS, Willard-Gallo K, Sangiolo D, Saba L. Immunotherapy Associated Pulmonary Toxicity: Biology Behind Clinical and Radiological Features. Cancers. 2019;11(3).

32. Samstein RM, Lee CH, Shoushtari AN, Hellmann MD, Shen R, Janjigian YY, Barron DA, Zehir A, Jordan EJ, Omuro A, Kaley TJ, Kendall SM, Motzer RJ, Hakimi AA, Voss MH, Russo P, Rosenberg J, lyer G, Bochner BH, Bajorin DF, Al-Ahmadie HA, Chaft JE, Rudin CM, Riely GJ, Baxi S, Ho AL, Wong RJ, Pfister DG, Wolchok JD, Barker CA, Gutin PH, Brennan CW, Tabar V, Mellinghoff IK, DeAngelis LM, Ariyan CE, Lee N, Tap WD, Gounder MM, D'Angelo SP, Saltz L, Stadler ZK, Scher HI, Baselga J, Razavi P, Klebanoff CA, Yaeger R, Segal NH, Ku GY, DeMatteo RP, Ladanyi M, Rizvi NA, Berger MF, Riaz N, Solit DB, Chan TA, Morris LGT. Tumor mutational load predicts survival after immunotherapy across multiple cancer types. Nature genetics. 2019;51(2):202-6. 
33. Liu L, Bai X, Wang J, Tang XR, Wu DH, Du SS, Du XJ, Zhang YW, Zhu HB, Fang Y, Guo ZQ, Zeng Q, Guo XJ, Liu Z, Dong ZY. Combination of TMB and CNA Stratifies Prognostic and Predictive Responses to Immunotherapy Across Metastatic Cancer. Clinical cancer research : an official journal of the American Association for Cancer Research. 2019;25(24):7413-23.

34. Lin A, Zhang J, Luo P. Crosstalk Between the MSI Status and Tumor Microenvironment in Colorectal Cancer. Frontiers in immunology. 2020;11:2039.

35. Rodriquenz MG, Roviello G, D'Angelo A, Lavacchi D, Roviello F, Polom K. MSI and EBV Positive Gastric Cancer's Subgroups and Their Link With Novel Immunotherapy. Journal of clinical medicine. 2020;9(5).

36. Schrock AB, Ouyang C, Sandhu J, Sokol E, Jin D, Ross JS, Miller VA, Lim D, Amanam I, Chao J, Catenacci D, Cho M, Braiteh F, Klempner SJ, Ali SM, Fakih M. Tumor mutational burden is predictive of response to immune checkpoint inhibitors in MSI-high metastatic colorectal cancer. Annals of oncology : official journal of the European Society for Medical Oncology. 2019;30(7):1096-103.

\section{Figures}


A

Disease Summary for CTHRC1

\begin{tabular}{|c|c|}
\hline Analysis Type by Cancer & $\begin{array}{l}\text { Cancer } \\
\text { vs. } \\
\text { Normal }\end{array}$ \\
\hline Bladder Cancer & \\
\hline Brain and CNS Cancer & 5 \\
\hline Breast Cancer & 20 \\
\hline Cervical Cancer & 1 \\
\hline Colorectal Cancer & 13 \\
\hline Esophageal Cancer & 5 \\
\hline Gastric Cancer & 9 \\
\hline Head and Neck Cancer & 7 \\
\hline Kidney Cancer & 2 \\
\hline Leukemia & 2 \\
\hline Liver Cancer & 2 \\
\hline Lung Cancer & 5 \\
\hline Lymphoma & 7 \\
\hline Melanoma & 1 \\
\hline Myeloma & \\
\hline Other Cancer & 6 \\
\hline Ovarian Cancer & 2 \\
\hline Pancreatic Cancer & 2 \\
\hline Prostate Cancer & 2 \\
\hline Sarcoma & \\
\hline Significant Unique Analyses & 91 \\
\hline Total Unique Analyses & 281 \\
\hline
\end{tabular}

$\square \square \square \square \square \square \square$

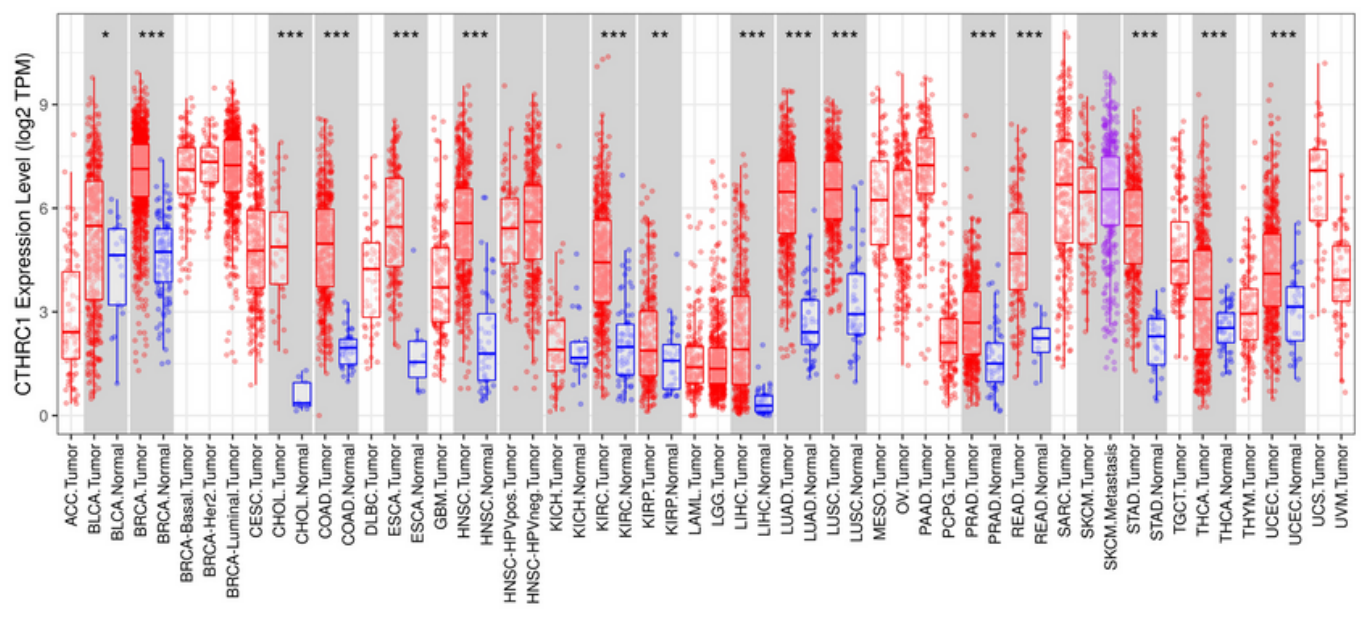

$\mathrm{C}$

Expression of CTHRC1 across TCGA cancers (with tumor and normal samples)
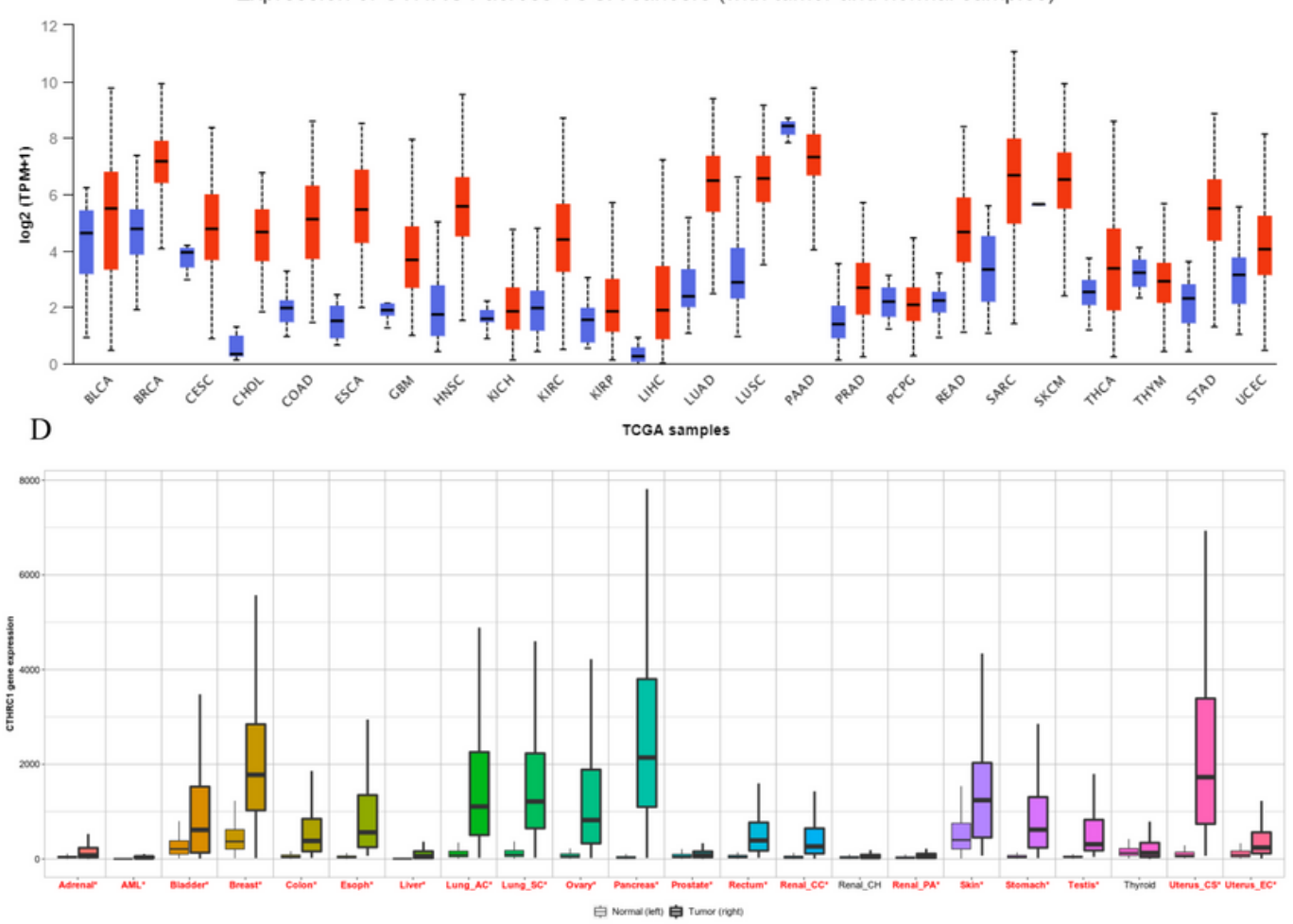

\section{Figure 1}

Transcript levels of CTHRC1 in tumor tissues. (A) Differential expression of CTHRC1 in human cancers and corresponding normal tissues analyzed by the oncomine database. (B) TIMER database analyzed CTHRC1 transcript levels in TCGA cancer and normal samples. (C) Box plot showing mRNA expression of CTHRC1 in cancer and non-cancer tissues analyzed by the UALCAN database. (D) CTHRC1 expression levels in multiple human cancers and matched normal tissue specimens analyzed by the TNM plot database. Note: $\mathrm{P}<0.001 / * \star \star, P<0.01 / * \star, \mathrm{P}<0.05 / *$. 
A ACC

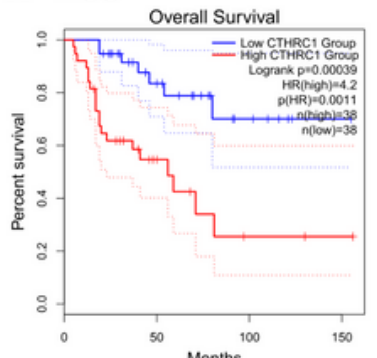

E KIRP

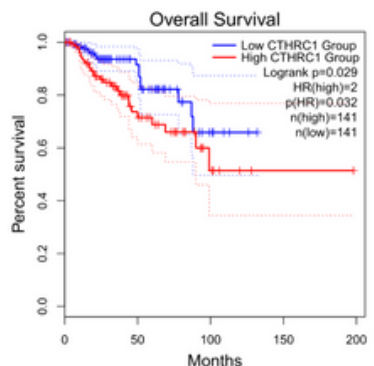

I LIHC

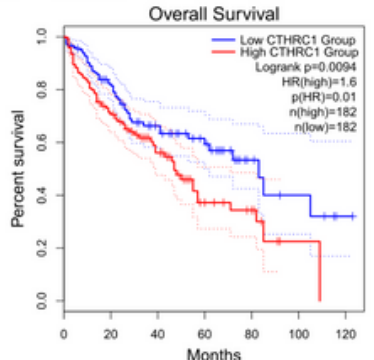

M KIRC Disease Free Survival

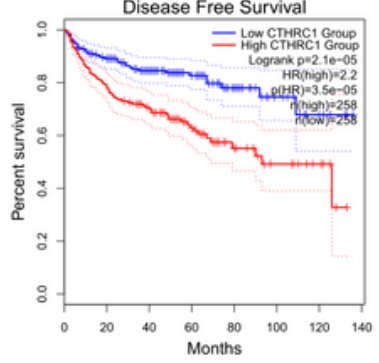

Q OV CTHRC1 (225681_at)

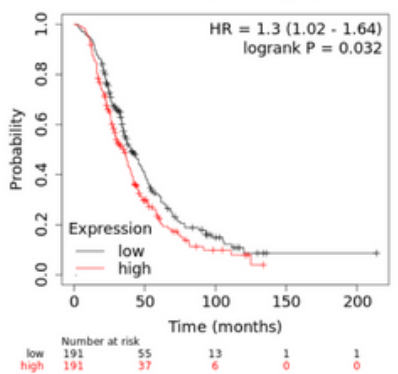

B ACC

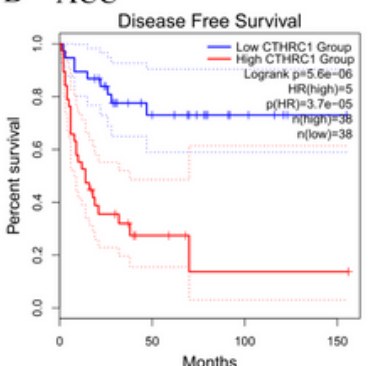

F KIRP

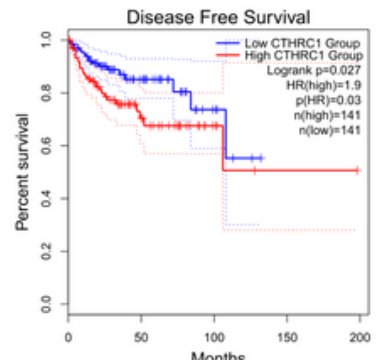

J SARC

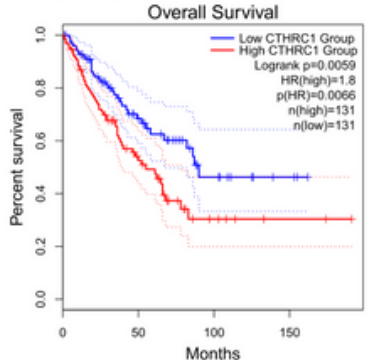

N PRAD

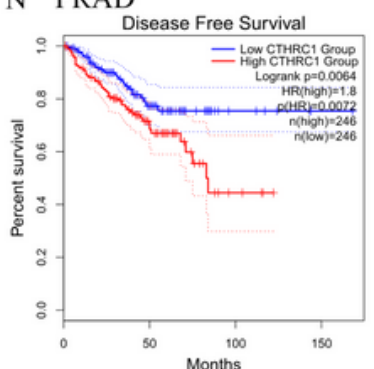

R BRCA

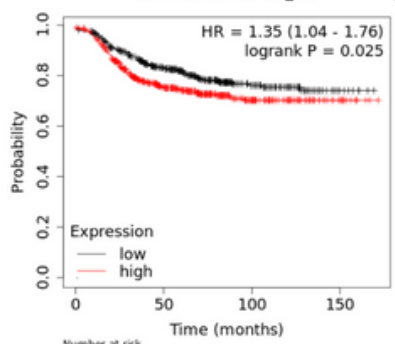

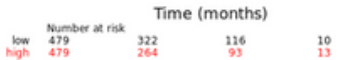

C COAD

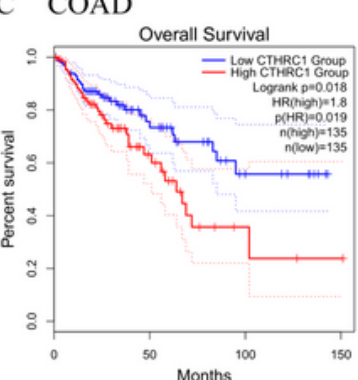

G BLCA

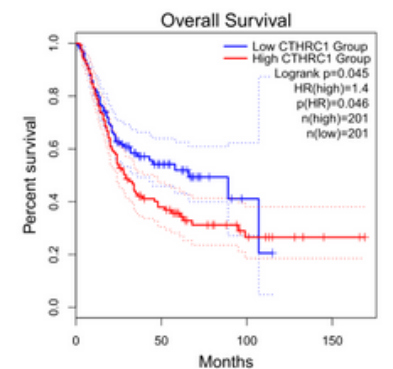

K STAD Overall Survival

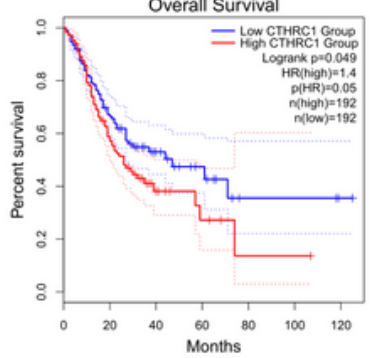

$\mathrm{O}$ OV CTHRC1 (225681_at)

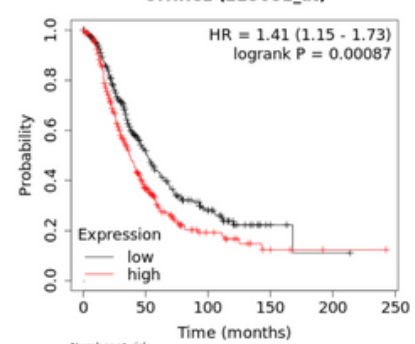

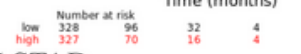

S STAD CTHRC1 (225681_at)

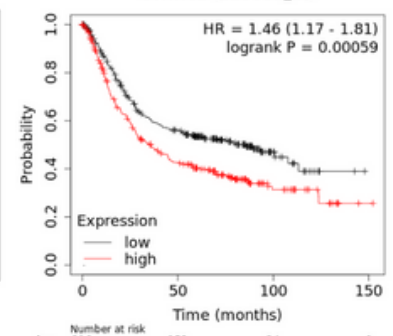

D COAD

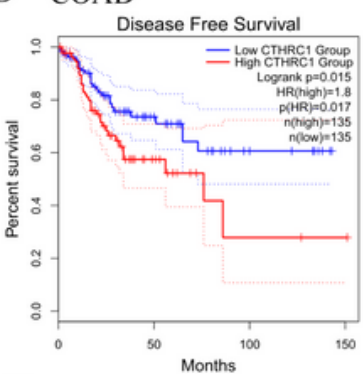

H LGG

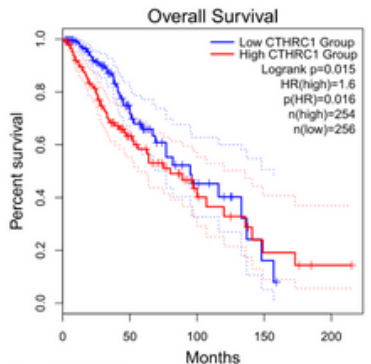

L $\mathrm{KICH}$

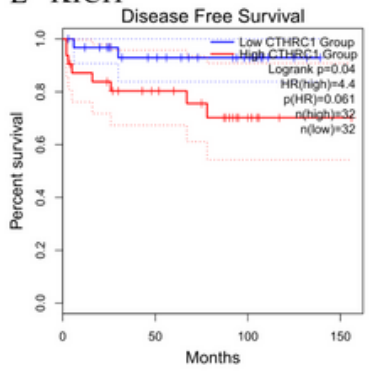

P OV cTHRC1 (225681_at)

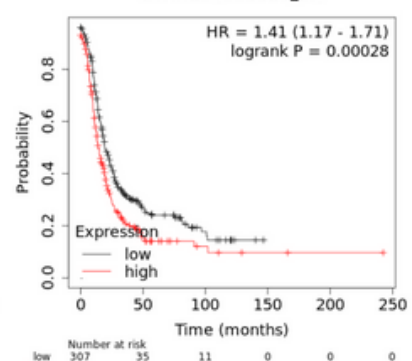

chand

T STAD crrRc1 (225681_at)

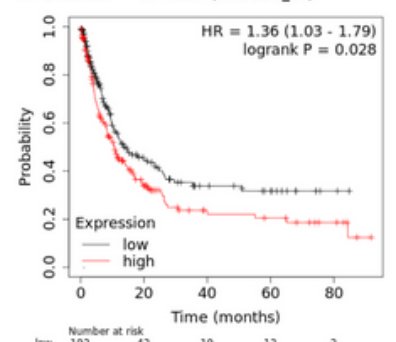

Figure 2

Association of CTHRC1 transcript levels with prognosis in cancer patients. (A-N) Analysis from GEPIA2.0 tool built based on TCGA RNA-seq data (A-F) High CTHRC1 transcript levels were associated with poor OS and DFS in ACC, COAD and KIRP. (G-K) There is a link between CTHRC1 mRNA overexpression and poor OS in BLCA, LGG, LIHC, SARC and STAD. (L-N) High expression of CTHRC1 was associated with poor DFS in $\mathrm{KICH}$, KIRC and PRAD. (O-R) Chip data from KM-PLOTTER database. (O-Q) High CTHRC1 
expression was related to worse OS, PFS and PPS in OV. (R) High CTHRC1 transcript levels were related to worse DMFS in BRCA. (S-T) For STAD patients, CTHRC1 was associated with worse OS and PPS. Note: OS/ overall survival; DFS/ disease free survival; PPS/ post progression survival; PFS/ progression free survival; DMFS/ distant metastasis free survival.
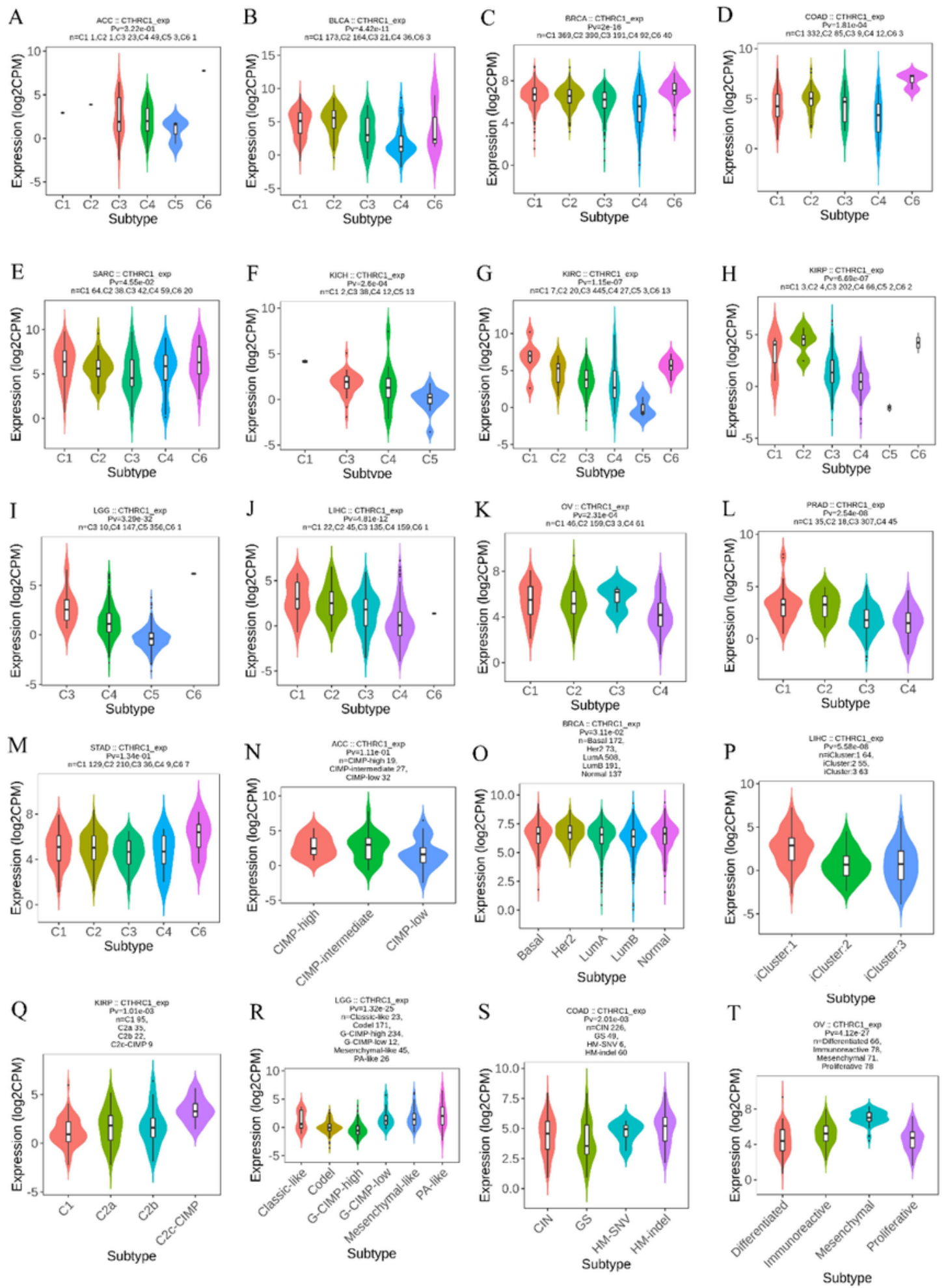

Figure 3 
The association of CTHRC1 with tumor immunologic and molecular typing analyzed by TISIDB. (A-M) The correlation between CTHRC1 transcription levels and immune typing in ACC, BLCA, BRCA, COAD, SARC, KICH, KIRC, KIRP, LGG, LIHC, OV, PRAD and STAD. (N-T) CTHRC1 mRNA expression was related to molecular classification in ACC, BRCA, LIHC, KIRP, LGG, COAD and OV.

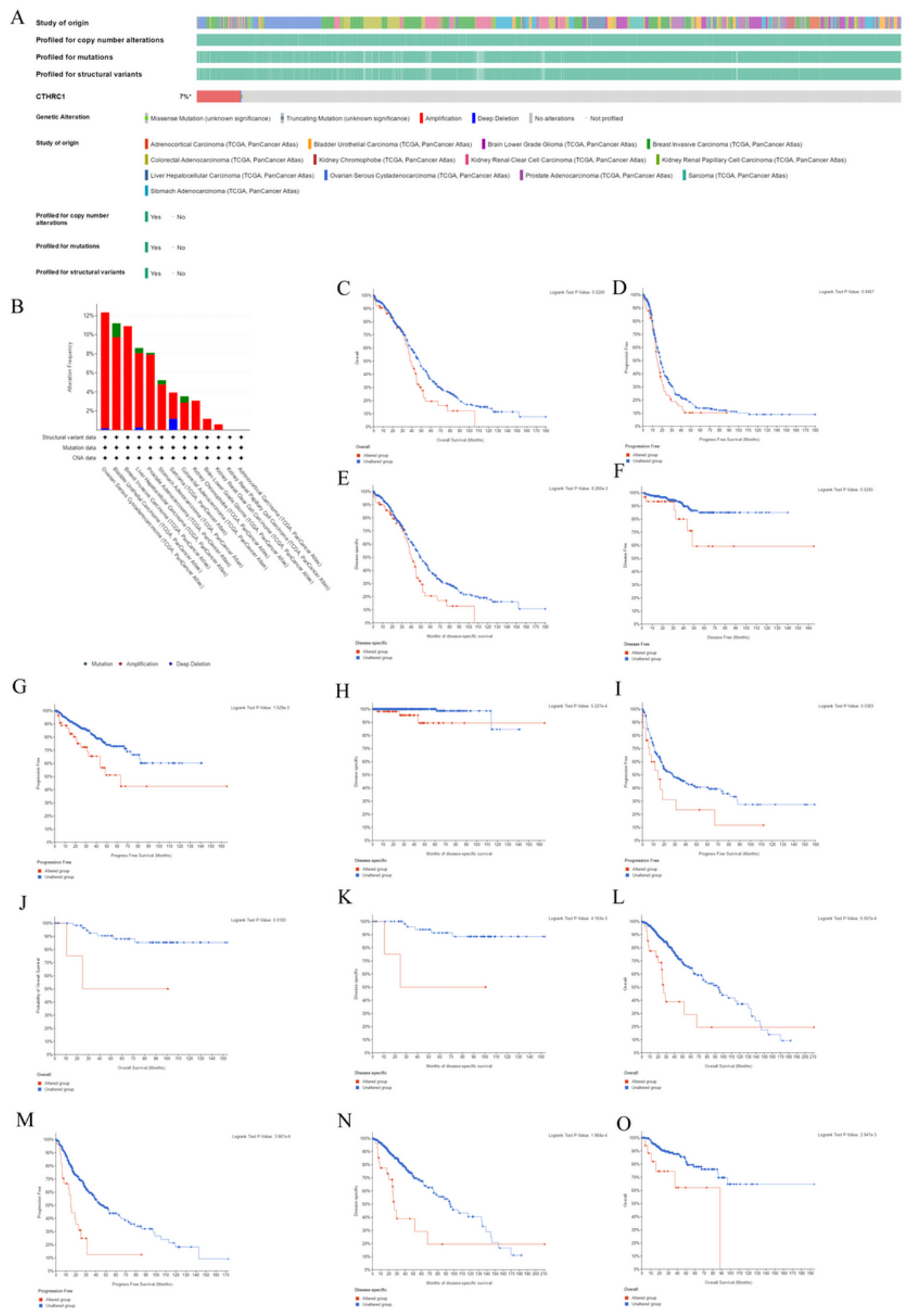

Figure 4 
Genomic alterations and prognosis of CTHRC1 in cancer tissues by using cBioPortal database. (A) Genomic alterations of CTHRC1 in 13 TCGA tumor samples (Different colors represent different types of genomic alterations: Red, amplification. Blue, deep deletion. Green, missense mutation. Gray, no alterations). (B) Specific information on the types of CTHRC1 genome alterations in 13 TCGA cancer cohorts. (C-E) The association of CTHRC1 genomic alterations with worse OS, PFS and DFS in OV. worse DFS, PFS and DSS in PRAD (F-H), worse PFS in SARC (I), worse OS, and DSS in $\mathrm{KICH}(\mathrm{J}-\mathrm{K})$, worse OS, PFS and DSS in LGG (L-N), worse OS in KIRP (O). Note: DSS/disease specific survival

A

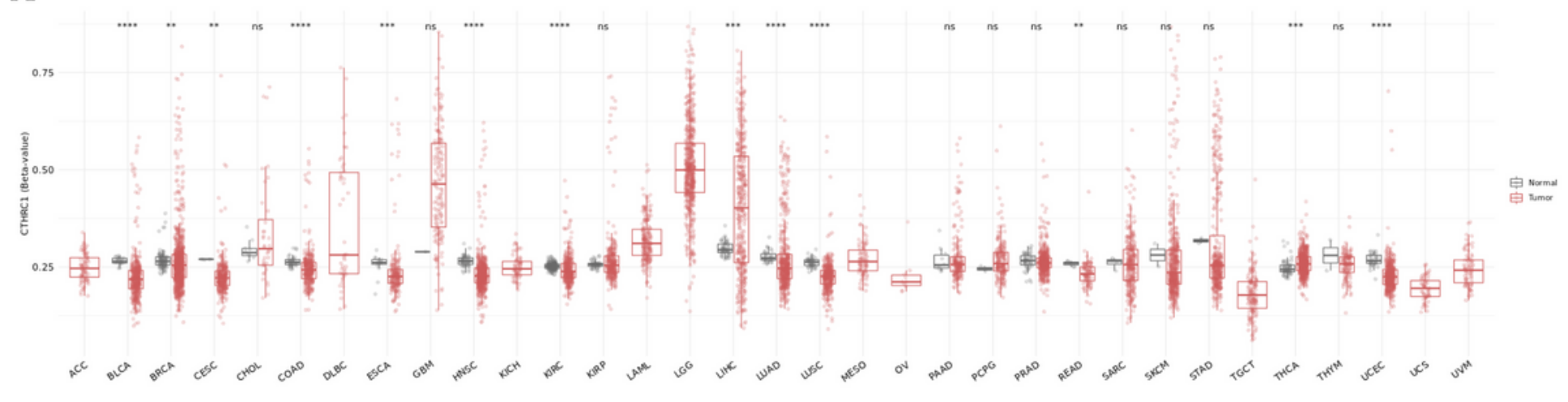

B

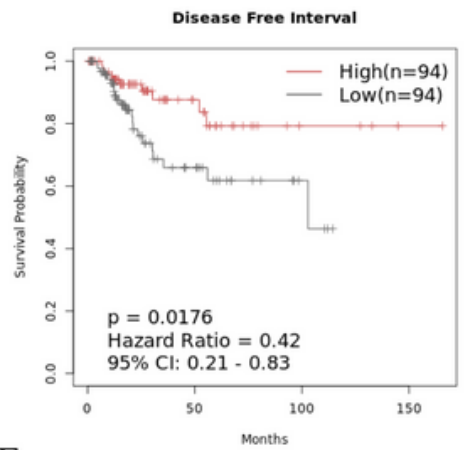

$\mathrm{F}$

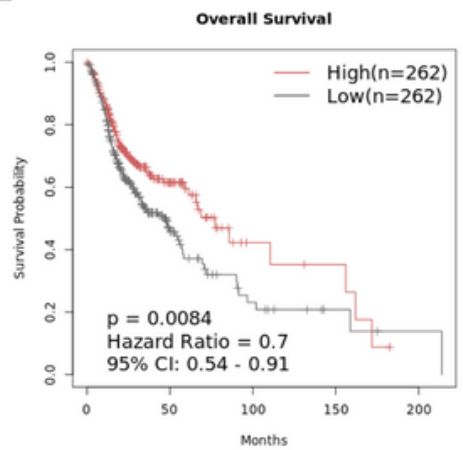

J

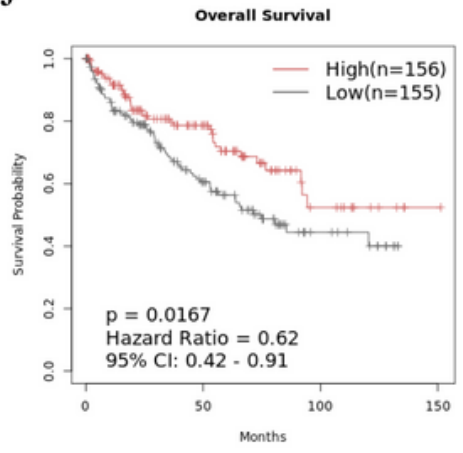

C

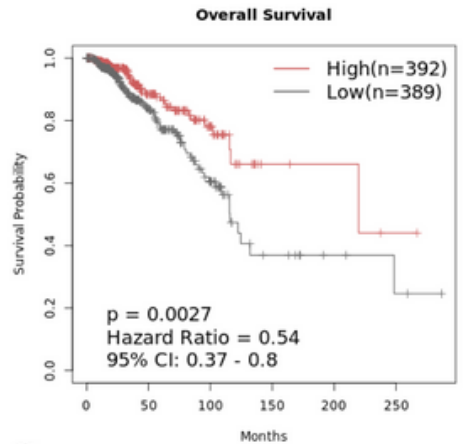

G

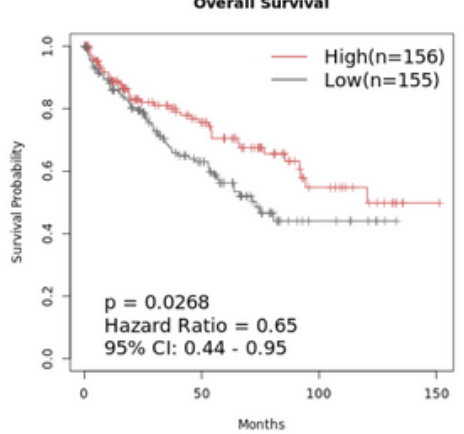

K

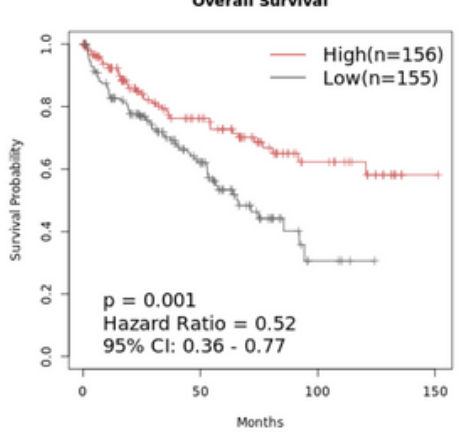

D

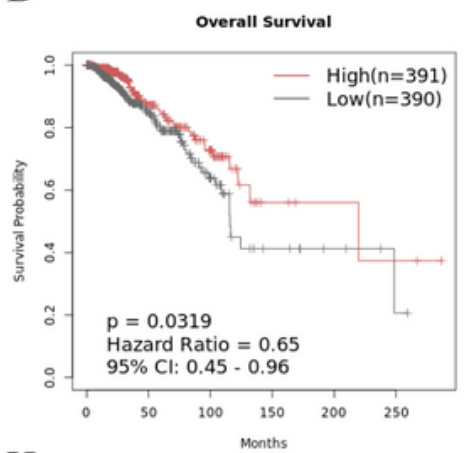

H

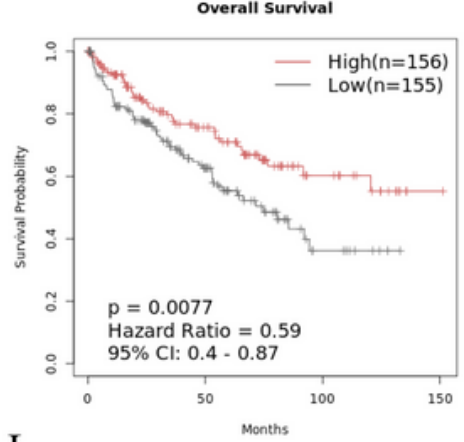

L

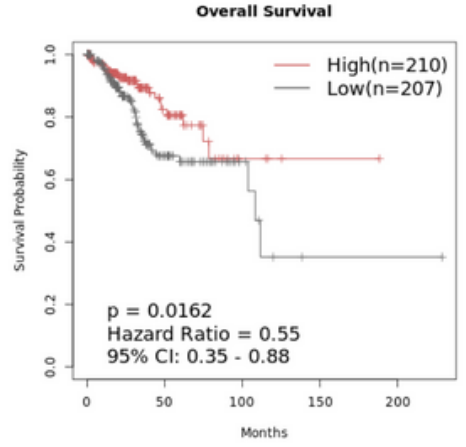

E

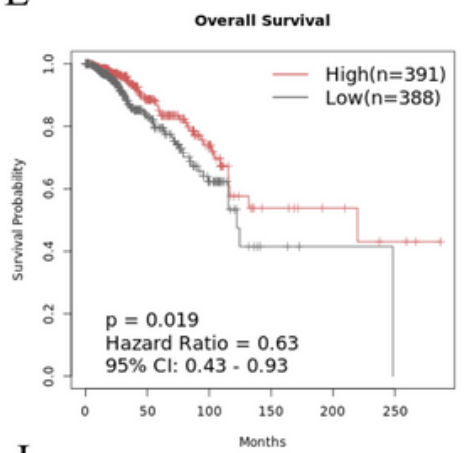

I

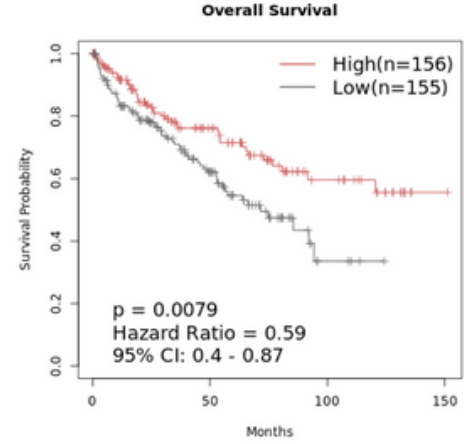

M

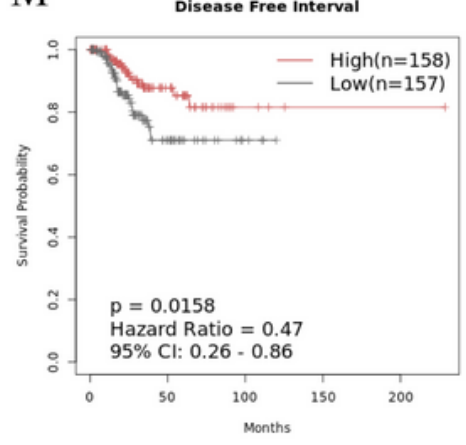




\section{Figure 5}

CTHRC1 methylation and prognosis in pan-cancer analyzed by SMART. (A) CTHRC1 methylation levels in cancer and matched non-cancer tissues. (B)The relationship between multiple methylation sites of CTHRC1 and prognosis in BLCA/ DFS/ cg01200376(B). BRCA/ OS/ cg11889769, cg17630389 and cg20447655 (C-E). HNSC/ OS/ cg20392240(F). KIRC/ OS/ cg01451193, cg04219247, cg11889769, cg19188612 and cg20392240(G-K). UCEC/ OS/ cg01200376(L). UCEC/ DFS/ cg17630389(M).

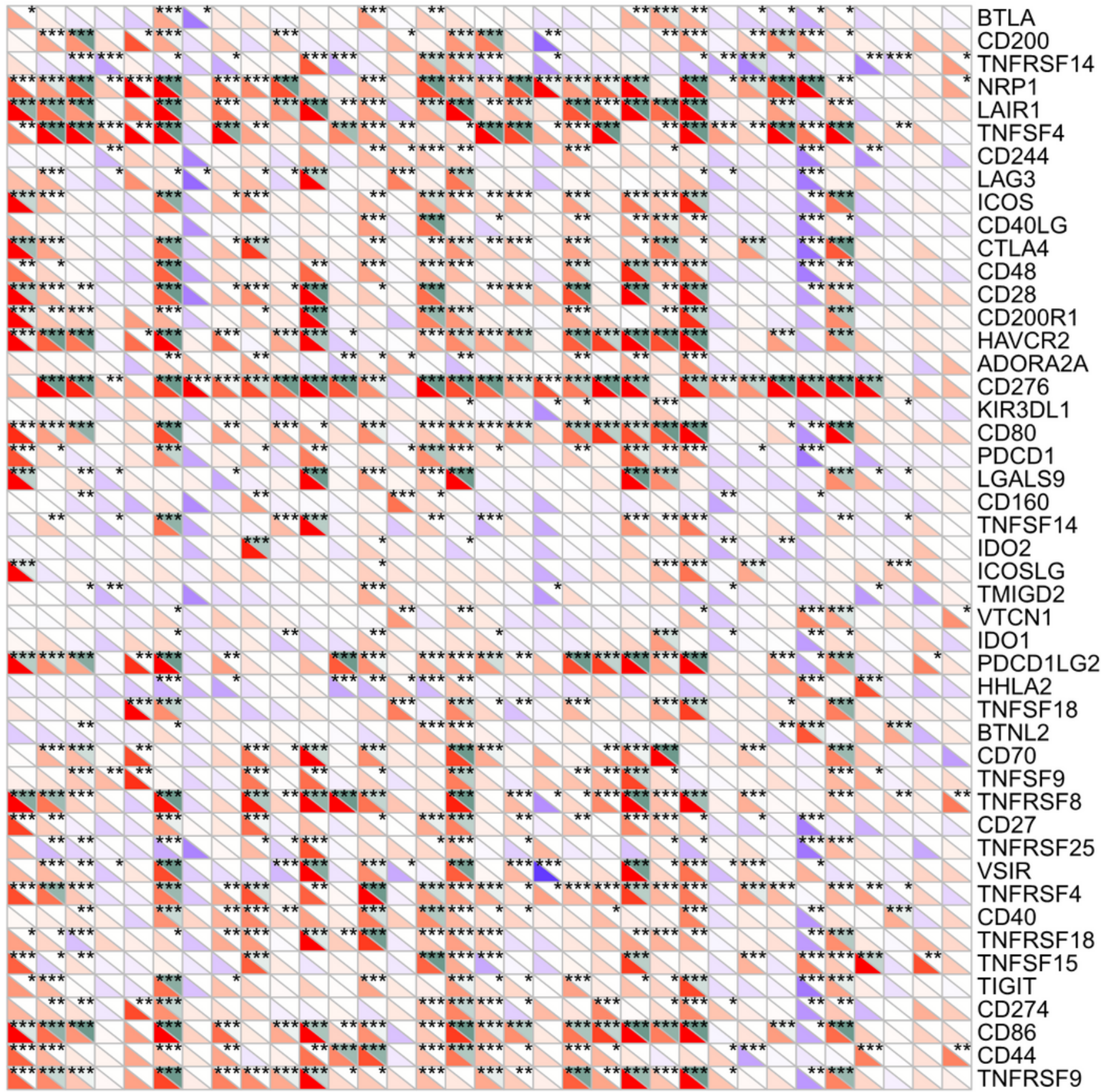

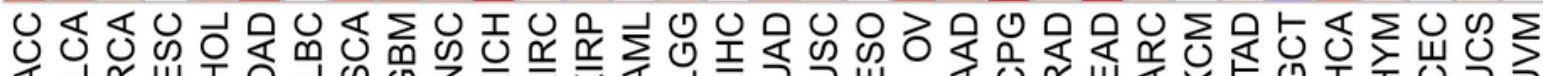

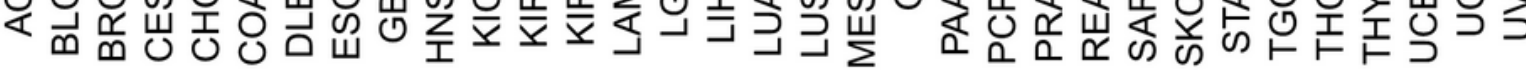

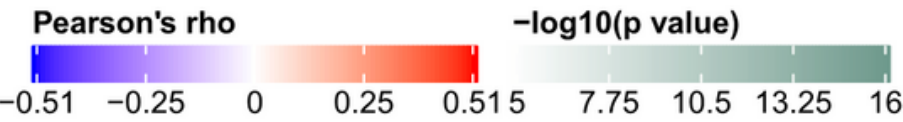


Figure 6

The correlation between CTHRC1 transcription levels and 50 immune checkpoints in pan-cancer.
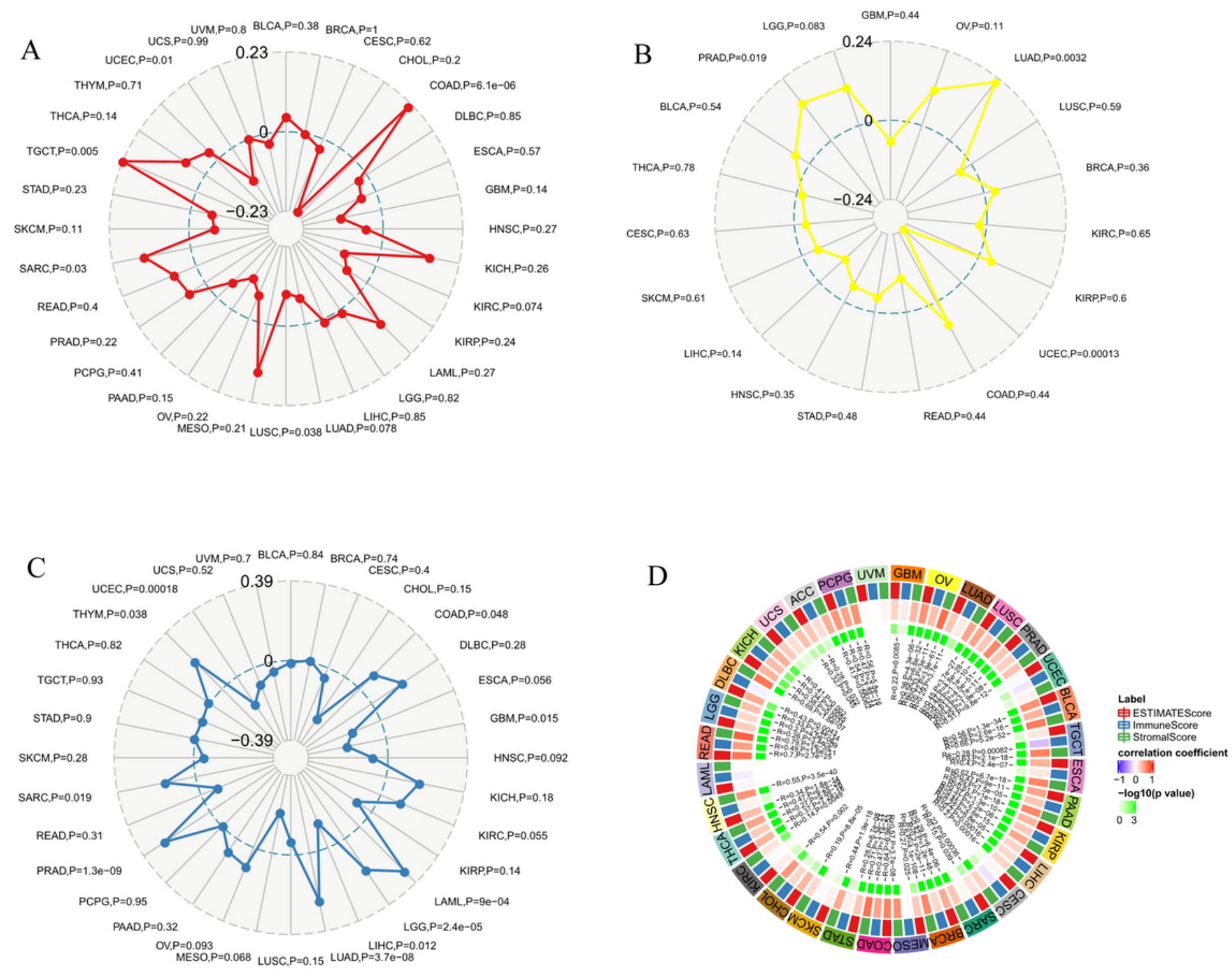

Figure 7

The Correlation between CTHRC1 transcription levels and MSI, neoantigen, TMB, and ESTIMATE Score in pan-cancer. Note: MSI: microsatellite instability, TMB: tumor mutational burden. 


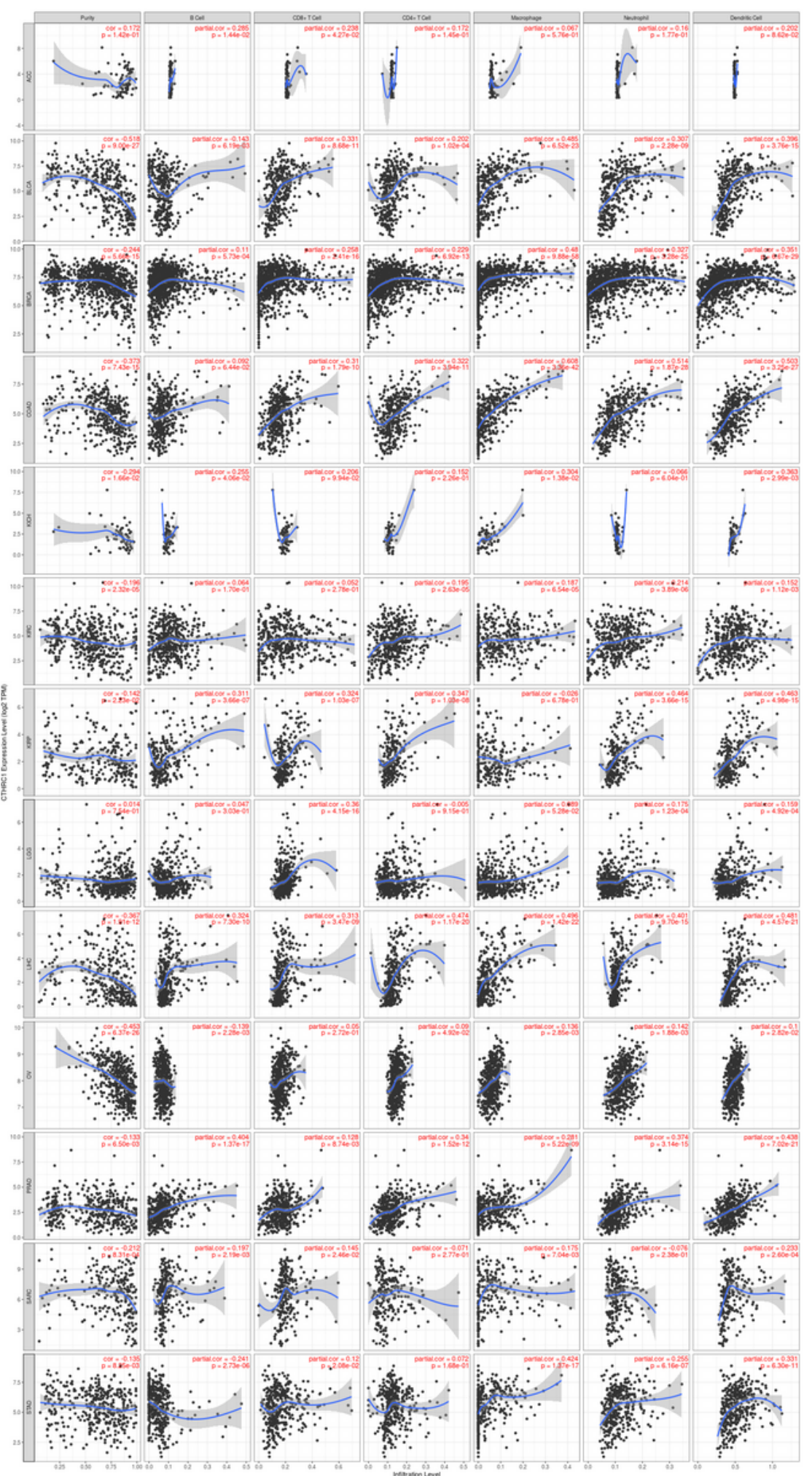

\section{Figure 8}

The association of CTHRC1 mRNA level with the number of immune cells in tumor tissues. 